\title{
A Short Pulse Laser System to Detect Concentration of Polychloro-Dibenzodioxine, -Furans
}

\author{
D.Q. Hoa ${ }^{1 *}$, N.D. Hoang ${ }^{1}$, T. Imasaka ${ }^{2}$ \\ ${ }^{1}$ Center for Quantum Electronics, Institute of Physics, VAST \\ 10 Dao Tan - Ba Dinh - Ha noi, Viet Nam \\ ${ }^{2}$ Department of Applied Chemistry, Kyushu Universtity, Japan \\ *e-mail: hoado@iop.vast.ac.vn
}

(Received: 29 June 2010; accepted: 13 September 2010)

\begin{abstract}
The method based on the multiphoton Ionization Mass Spectroscopy/Supersonic Jet (MPI-MS/SSJ) technique for determination of trace concentration of toxic organic substances in the air is studied and applied in many laboratories in the world. A short pulse laser system with Fourier transform - limited bandwidth is suitable to the alternatively ionization process of the cold molecules. The Distributed Feedback Dye Laser (DFDL) pumped by a Nd:YAG picosecond laser is setup and employed to this purpose. The short laser pulse of $12 \mathrm{ps}$, which is exactly adjusted to wavelength of $316.14 \mathrm{~nm}$ and $315.83 \mathrm{~nm}$ with bandwidth of $0.05 \mathrm{~nm}$, is corresponding to detect polychlorodibenzofuran and polychlorodibezodioxine
\end{abstract}

Key words: MPI-MS/SSJ technique, short laser pulse, concentration detection

\section{INTRODUCTION}

Every day gas exhausted from incinerator, transportation means or industry used fossil energy discharge to the environment many kinds of pollutants containing polychlorinated biphenyl (PCB), polychlorinated dibenzodioxins (PCDDs), and polychlorinated dibenzofurans (PCDFs) [1]. Because of dangerous toxicity of these substances a method to detect trace concentration in the environment is necessarily required. As it is well known, the dioxin molecules are highly toxic not only for two benzene rings in molecular structure but also for the chlorination of the molecule. It notes that the toxicity of dioxin or furan depends on both the number of chlorine ion and position of chlorine element in the structure $[2,3]$. Therefore, it is difficult to detect these molecules by using conventional equipments such as gas chromatograph (GC) or mass spectroscopy (MS).

Up to now, the gas chromatography method combined to mass spectroscopy technique (GC/MS) for detection of trace concentration of this class has been applied in many laboratories in the world. However, one has to spend a long time and much money to receive the result of one sample, e.g. one week and 3000 US\$ in Japan. Super sonic jet (SSJ) technique could provide single electronic energy states, which were almost removed the rot-vibration energy states, due to low temperature that appeared in the working space, c.a. less than $10 \mathrm{~K}$. Assuming that the molecules in the working space were alternatively ionized, the sensitivity of the GC/MS method should be significantly improved. The method relied on multi photon ionization/supersonic jet/mass spectroscopy (MPI/SSJ/MS) is a good tool to monitor chlorinated dioxin or furan isomers. In fact, electronic energy state of these molecules has a variety to be just the same as vibration energy states. The application of a narrowband, short pulse, and tunable laser for the ionization source is considered a desirable solution in order to enhance the equipment effect. A short pulse laser using quenched distributed feedback dye laser (DFDL) [6] is possible to employ that purpose. This is a kind of double oscillator with a common gain medium. The quenching effect of second cavity, which is arranged such certain delay time that DFDL's starting, could remove second pulses of DFDL.

In this report, we present several new approaches of the research on a picosecond high monochromatic, continu- 
ously tunable laser, in which a picosecond Nd:YAG is applied as a pumping source. The application of this laser system for detection of polychlorinated dibenzodioxin and polychlorinated dibenzofurans is demonstrated.

\section{DEVELOPMENT OF A SINGLE PULSE PICOSECOND LASER}

A dye laser based on the distributed feedback (DFB) principle has been studied and developed for many years $[4,5]$. An important aspect of DFB structures is that the grating period $(\Lambda)$ of the periodic index or gain pattern sets the laser emission wavelength following the relation $\lambda=2 \Lambda n_{\text {eff }} / m$ where $n_{\text {eff }}$ is the effective refractive index of the guided mode responsible for lasing and $m$ is the order of diffraction responsible for the feedback. Thus, the laser emission wavelength may be tuned within the gain spectrum of the medium (typically $\sim 40 \mathrm{~nm}$ ) by altering either $\Lambda$ or $n_{\text {eff }}$, while the gain spectrum can be displaced throughout the visible and near infrared with the use of different laser dyes. On the other hand, a single picosecond laser pulse could be extracted from the series of picosecond laser pulse by using another picosecond laser to pump into the DFB oscillator.

The diagram for the experiment is shown in Fig. 1. A picosecond Nd:YAG laser (Continuum, Leopard, 120 ps, $10 \mathrm{~Hz}$ ) is employed as a pumping source of the dye laser. The oscillator of DFB is a cell of $3 \times 3 \mathrm{~mm}$ contained circulation mixed dye solution (RhB/DCM) in PC (propylene carbonate) or methanol solvent with concentration of $3 \times 10^{-3} \mathrm{M} / \mathrm{L}$ for $\mathrm{DCM}$ and $1 \times 10^{-4} \mathrm{M} / \mathrm{L}$ for $\mathrm{RhB}$. The pulse energy of $180 \mu \mathrm{J}$ of second harmonic generation of $\mathrm{Nd}$ :YAG laser divided to two beams by a couple mirror is focused onto a dye cell formed a well known DFB oscillator [4]. The output pulse is amplified in three amplifier stages with two stages of side pumping and post amplifier of quasi-longitudinal pumping. Two preamplifiers contained $1 \times 10^{-4} \mathrm{M} / \mathrm{L}$ circulation $\mathrm{RhB} / \mathrm{DCM}$ dye solution used in laser configuration can be explained as follow: For efficient power extraction from an amplification medium, the seed pulse needs to have certain intensity, close to the saturation fluence. The seed pulse before amplification is only hundreds $\mathrm{nJ}$, and can only extract energy if it is focused to a relatively small diameter. In order to get enough gain in the first stage it is actually "over-pumped" meaning that significant amounts of ASE are generated, comparable to the energy of the amplified pulse. Using two pre-amplifiers, the amplified pulse is now sufficiently intense to compete with ASE in the final stages. Thus, ASE emission is decreased in laser beam. Corresponding to pulsewidth of pumping source (120 ps), the suitable gain of amplified medium is shortly attained. A quasi-longitudinal configuration of the final amplifier is selected to increase the amplified effect. The gain is varied in amplification medium along the pumping direction in the same beamline of DFB laser, so this allows the maximum gain extraction contained in the post amplifier. The advantage of typical post amplifier in picosecond pumping configuration is shown by reducing ASE contribution (c.a. $5 \%$ ) in laser output. The UV beam is approached by second harmonic generation of fundamental beam using a BBO crystal $6 \times 6 \times 5 \mathrm{~mm}$ (VLOC Co.). The characters of DFB laser is monitored by a wavemeter (WA 5500, Burleigh), an autocorrelator, and microjoule meter (molectron, M3-J5).

The temporal and bandwidth properties of the DFB are shown in Fig. 2. A laser bandwidth of $8.2 \pm 0.2$ ps is observed using an autocorrelator. The bandwidth is strongly dependent on pumping rate [7]. In the case of high pumping energy, the pulse profile is sometimes modified to

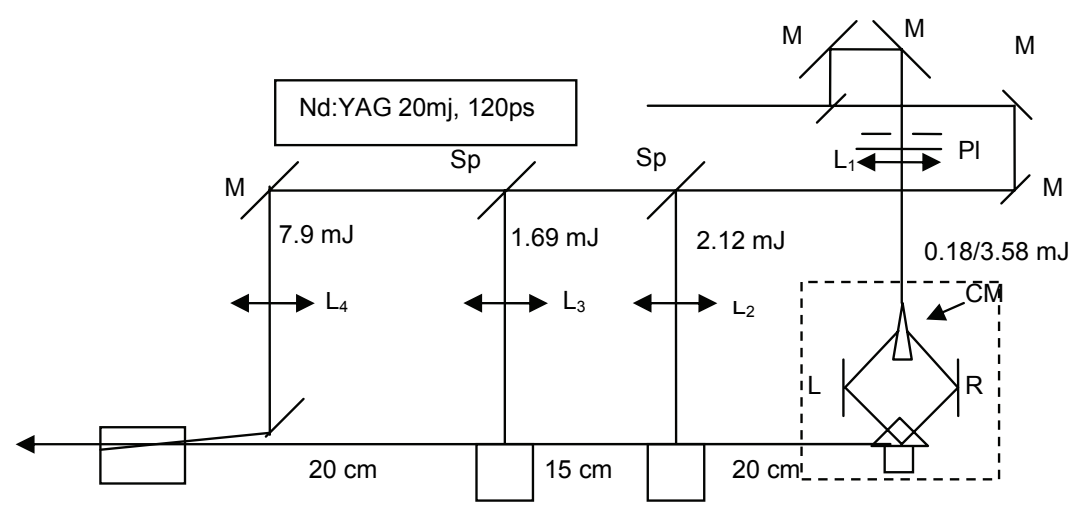

Fig. 1. Laser dye: DCM of concentration of $1 \times 10^{-3} \mathrm{M} / \mathrm{L}$ (osc), $1 \times 10^{-4}$ (1-st amp) and $0.5 \times 10^{-4}$ (final amp) in methanol (quality is rapidly decayed) and polypropylene carbonate; CM: Beam divider by couple mirror; $L_{1}$ : cylindrical lens $(f=300 \mathrm{~mm}) ; L_{2}, L_{3}$ : cylindrical lens $(f=100 \mathrm{~mm}) ; L_{4}$ : convex lens $(f=500 \mathrm{~mm})$ 
show some structures on the envelope. At pump power closed to 2 times above threshold, this structure is inexplicitly displayed. The short pump effect almost removes subpulses in profile of DFB laser. The pulse shape can be easily predicted as limited Fourier-transform of an entire spectrum. The product of pulsewidth and linewidth of DFB laser pulse attained 0.441 for Gaussian shape is obtained. This laser is suitable for application of MPI/SSJ/MS method.

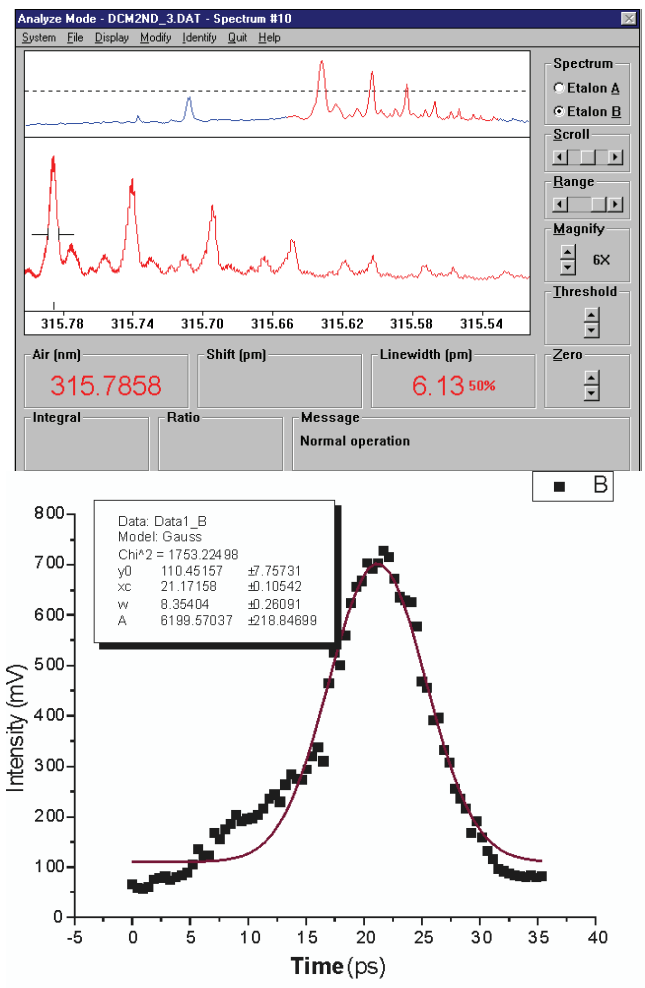

Fig. 2. Properties of the DFB laser using laser dye of DCM, (a) bandwidth of laser from wavemeter, and (b) pulse width using an autocorrelator

\section{MASS SPECTROSCOPY OF PCDD/F}

The concentration of $\mathrm{PCDD} / \mathrm{F}$ in the air is very low, ca. few ppb [8]. The mass spectra of the sample with study substances are recorded by using the system shown in Fig. 3. The laser beam from DFB laser with active medium of DCM dye provided a tunable range of 592-650 $\mathrm{nm}$ is multiplied frequency by a non-linear BBO crystal. The UV emission separated from mixing beam by a UV filter is directed to a certain position into jet sample space through a quartz window fixed on a vacuum chamber. The trigger signal is collected from a photodiode (Hamamatsu S3399, 100MHz) placed on an outline window of the vacuum chamber. Vaporized analyte was entrained into argon carrier gas, which is expanded from a pulse nozzle (General Valve Inc., GV9279-900, $200 \mu \mathrm{s}$ ) and was localized in the jet. An MPI spectrum was recorded by monitoring the molecular ion peak using a boxcar integrator. For recording mass spectrum, 200 transient signals were measured and accumulated using digital oscilloscope (Lecroy, 9360). Among all penta-CDF isomers, the concentration of some isomers decides the toxicity level, for example 1,2,3,8,9- penta-CDF less toxic than $1,2,3,7,8$ penta-CDF. Therefore, we set up the experiment to detect this isomer.

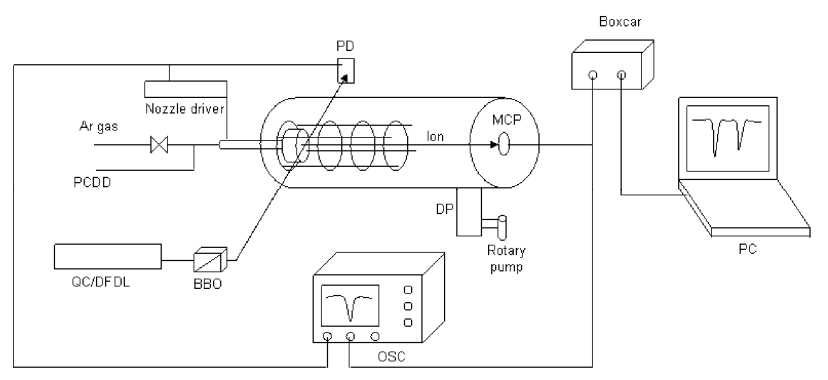

Fig. 3. Experimental setup of MPI/SSJ/MS to detect chlorodibezofurans

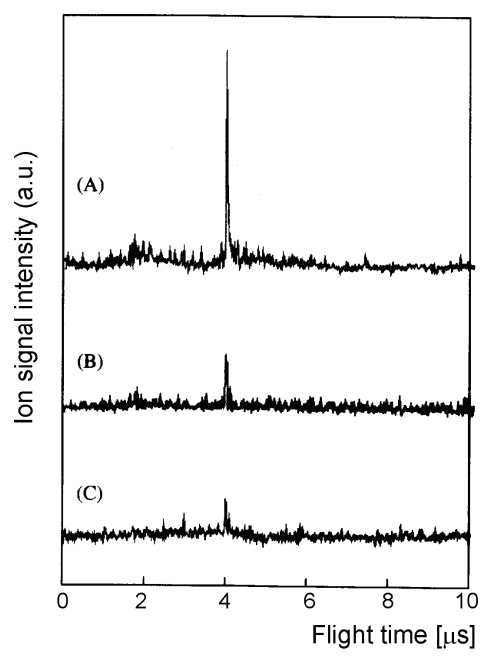

Fig. 4. Time of flight of 2,3,4,7,8-pentaCDF ion

The laser wavelength of $315.83 \mathrm{~nm}$ as the ionization source for 2,3,4,7,8-pentaCDF is selected. The result of the mass spectrum of pentachlorodibenzofuran is displayed in Fig. 4. The ratio of signal to noise is decreased to 7-folds using the narrow band picosecond pulse laser as the ionizing source of molecules. The ion signals in the mass spectrum increase with increasing pulse energy in experiments using either picosecond or nanosecond lasers. However, the molecular ions are more easily dissociated to small fragments at higher pulse energy. This efficient dissociation under a nanosecond laser pulse is well explained by the 
model based on the ladder-switching mechanism given by Yang and co-workers [9]. Thus, ionization efficiency can be improved 7-times by replacing a nanosecond laser with a transform-limited bandwidth picosecond laser. The result shown in Fig. 4 suggests that picosecond multipoton ionization is suitable for detection of a molecular ion.

\section{CONCLUSION}

Multiphoton ionization mass spectral technique is an ideal tool to detect the isomers of polychlorodibenzofurans or polychlorodibenzodioxin. By using the picosecond laser with Fourier transform-limited bandwidth, the ionization effect of these molecules is really enhanced. Experimental observed results are reasonable to published theoretical works. The short pulse laser technique based on distributed feedback dye laser to satisfy technique requirements is demonstrated.

\section{References}

[1] F. Muhlberger, R. Zimmermann, A. Kettrup, Anal. Chem. 73, 3590 (2001).

[2] R. Zimmermann, H.J. Heger, A. Ketrup, U. Boesl, Rapid Comm. Mass Spectrom. 11, 1095 (1997).

[3] T. Uchimura, T. Imasaka, Anal. Chem. 72, 2648 (2000).

[4] P.P. Yaney, D.A. Kliner, P.E. Schrader, R.L. Farrow, Rev. Sci. Instrum. 71, 1296 (2000).

[5] C. Lynga, F. Ossler, T. Metz, J. Larsson, Appl. Phys. B72, $913(2001)$

[6] D.Q. Hoa, N. Takeyasu, T. Imasaka, N. Dai Hung, Rev. Sci. Instrum. 74, 28 (2003).

[7] D.Q. Hoa, V. Duong, P. Long, T. Imasaka, T.H. Nhung, JKPS 3823 (2008).

[8] T. Onoda, G. Saito, T. Imasaka, Anal. Chim. Acta 412, 213 (2000).

[9] J.J. Yang, D.A. Gobeli, M.A. El-Sayed, J. Phys. Chem. 89, 3426 (1985).
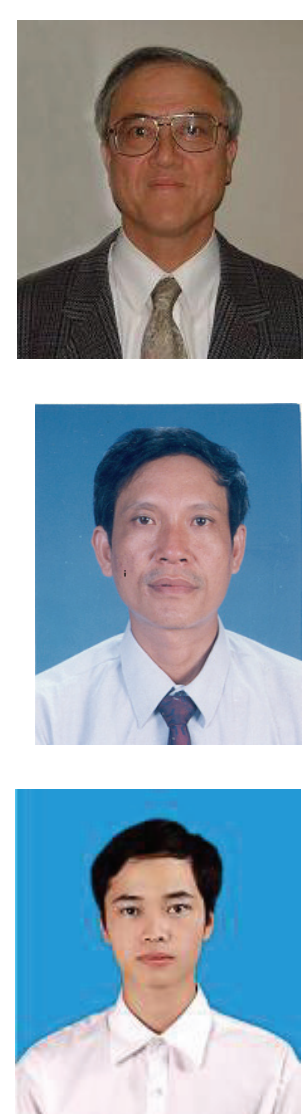

Totaro Imasaka, Professor of Applied Chemistry in Graduate School of Engineering, Kyushu University, was born in 1950 in Fukuoka. After receiving his Bachelor (1973), Master (1975), and Doctor (1978) degrees, he worked as a postdoctoral fellow at Stanford University. He returned to Kyushu University as Assistant Professor (1979), and subsequently promoted to Lecturer (1980), Associate Professor (1981), and Professor (1991). His current interests in research are gas chromatography combined with femtosecond laser ionization/mass spectrometry for the trace analysis of dioxins in the environment, and generating an ultimatelyshort optical pulse and a train of fastest optical pulses based on four-wave Raman mixing, referred to as "Rainbow Stars", which was accidentally found during the process of analytical spectroscopy in his laboratory. He is training himself by swimming and reading novels for relaxation.

Do Quang HoA, Principal researcher of Institute of Physics, was born in 1952 in Hanoi. After leaving from Army (1976), receiving his Bachelor (1980), he worked as a researcher in Institute of Geology (1981), and in Institute of Physics (1998). He awarded Doctor (2004) of Kyushu University of Japan. His interests in research are development of narrowband tunable short pulse laser system, application of the laser to analytical spectroscopy, and use of the short pulse laser to measure material properties in temporal field such as fluorescence lifetime, time resolution etc.

NGUYen Dinh Hoang, Master student of Institute of Physics, was born in 1985 in Hanoi. He received Bachelor in 2007 from College of technology, Vietnam National University, Hanoi. From 2007 to 2009, he was a member of Photonics laboratory, Institute of Physics, Vietnam Academy of Science and Technology. Now, he is a Master student of Institute of Physics, Vietnamese Academy of Science and Technology in laser spectroscopy field. 